\title{
An Efficient Algorithm for Calculating the Heat Capacity of a Large-scale Molecular System
}

\author{
Chao Yang * \\ National Energy Research Scientific Computing Center \\ Lawrence Berkeley National Laboratory \\ Berkeley, CA 94720 \\ cyang@lbl.gov \\ Donald W. Noid ${ }^{\dagger}$ and Bobby G. Sumpter ${ }^{\dagger}$ \\ Chemical \& Analytical Sciences Division \\ Oak Ridge National Laboratory \\ Oak Ridge, TN 37831-6197 \\ dwn@ornl.gov zbs@ornl.gov \\ Danny C. Sorensen $\ddagger$ \\ Department of Computational \& Applied Mathematics \\ Rice University \\ Houston, TX 77005 \\ sorensen@caam.rice.edu \\ Robert E. Tuzun ${ }^{\S}$ \\ Department of Computational Science \\ State University of New York at Brockport \\ Brockport, NY 14420 \\ rtuzun@brockport.edu
}

\footnotetext{
${ }^{*}$ This work was supported by the Director, Office of Science, Division of Mathematical, Information, and Computational Sciences of the U.S. Department of Energy under contract number DE-AC03-76SF00098. This research used resources of the National Energy Research Scientific Computing Center, which is supported by the Office of Science of the U.S. Department of Energy.

${ }^{\dagger}$ Research sponsored by Division of Material Sciences, Office of Basic Energy Sciences, U.S. Department of Energy under contract DE-AC05-00OR22725 with UT-Battelle, LLC.

${ }^{\ddagger}$ This work is supported in part by NSF Grant CCR-9988393 and by NSF Grant ACI-0082645.

${ }^{\S}$ Research supported by Petroleum Research Fund administered by the American Chemical Society.
} 


\begin{abstract}
We present an efficient algorithm for computing the heat capacity of a large-scale molecular system. The new algorithm is based on a special Gaussian quadrature whose abscissas and weights are obtained by a simple Lanczos iteration. Our numerical results have indicated that this new computational scheme is quite accurate. We have also shown that this method is at least a hundred times faster than the earlier approach that is based on estimating the density of states and integrating with a simple quadrature formula.
\end{abstract}

\title{
1 Introduction
}

The heat capacity of a large molecular system is defined by

$$
C_{v}=k_{B} \int_{0}^{\infty} \frac{\left(h \omega c / k_{B} T\right)^{2} e^{-h \omega c / k_{B} T}}{\left(1-e^{-h \omega c / k_{B} T}\right)^{2}} g(\omega) d \omega
$$

where $k_{B}$ is Boltzmann's constant, $c$ is the speed of light, $h$ is Planck's constant, and $\omega$ is the fundamental vibrational frequency in $\mathrm{cm}^{-1}[11]$. The function $g(\omega)$, often known as the density of states, describes the distribution of the vibrational frequencies of the particle. To be specific, $g(\omega) d \omega$ gives the number of vibrational frequencies within the interval $(\omega, \omega+d \omega)$. This definition yields a (cumulative) distribution function [5, p. 22]

$$
\sigma(\omega)=\int_{0}^{\omega} g(t) d t
$$

Since molecular systems are discrete, the integration in (1) shall be interpreted in the sense of Riemann-Stieltjes' integral [6, p. 64]. Naturally, the distribution function can be expressed by

$$
\sigma(\omega)=\sum_{i=1}^{n} h\left(\omega-\omega_{i}\right),
$$

where $h(\omega)$ is the Heaviside function

$$
h(\omega)= \begin{cases}1 & \omega \geq 0 \\ 0 & \text { otherwise }\end{cases}
$$

and $n$ is the total number of fundamental vibrational frequencies. Note that $n=3 m$, where $m$ is the number of atoms within the system.

It follows from the relation

$$
g(\omega)=\frac{d \sigma(\omega)}{d \omega}
$$

that $g(\omega)$ is simply a sum of $\delta$-functions. i.e.,

$$
g(\omega)=\sum_{i=1}^{n} \delta\left(\omega-\omega_{i}\right) .
$$


Clearly, the measure corresponding to the integration in this case is the point measure $\left\{\mu_{i}=1\right\}$, $i=1,2, \ldots, n$, defined on the fundamental frequencies $\left\{\omega_{i}\right\}$. It can be extended to any nonnegative point measure $\left\{\mu_{i}\right\}$ such that $\sum_{i=1}^{n} \mu_{i}=n$. Under this general definition, we have

$$
\sigma(\omega)=\sum_{i=1}^{n} \mu_{i} h\left(\omega-\omega_{i}\right), \text { and } g(\omega)=\sum_{i=1}^{n} \mu_{i} \delta\left(\omega-\omega_{i}\right) .
$$

For convenience, let us normalize the point measure such that $\sum_{i}^{n} \mu_{i}=1$. It follows that

$$
\int_{0}^{\infty} \sigma(\omega) d \omega=\sum_{i=1}^{n} \mu_{i} h\left(\omega-\omega_{i}\right)=1
$$

In Figure 1, we plot the distribution function of a 3000-atom polyethylene (PE) particle constructed in earlier studies [1]. The point measure used in the plot is $\mu_{i}=1 / n$ for $i=1,2, \ldots, n$. Using this measure, the heat capacity can be evaluated by

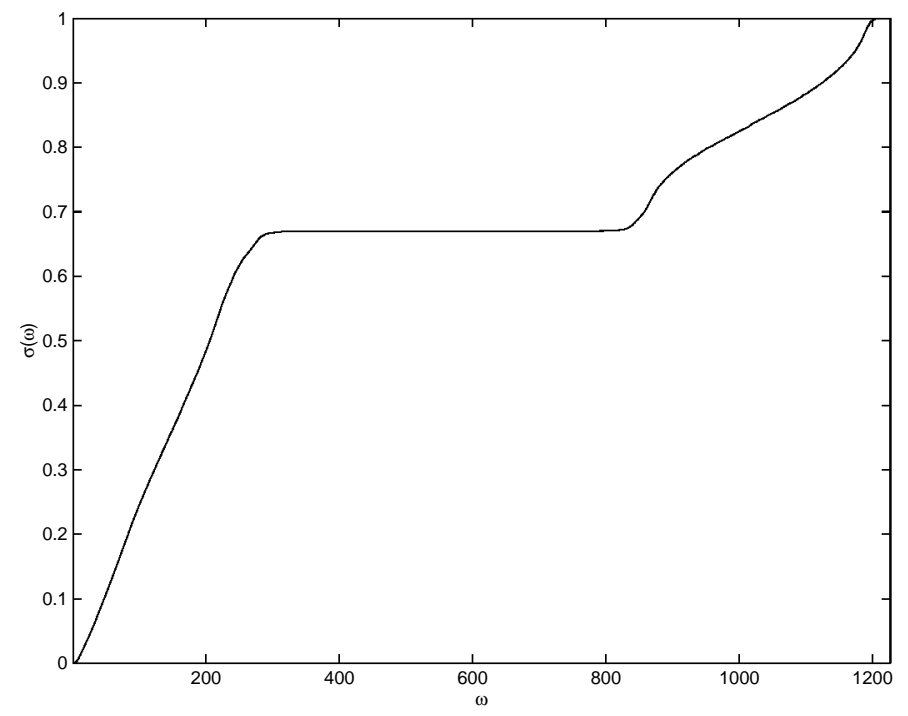

Figure 1: The distribution function for a 3000-atom PE particle.

$$
C_{v}=\sum_{i=1}^{n} H\left(\omega_{i}\right) / n
$$

where

$$
H(\omega)=k_{B} \frac{\left(h \omega c / k_{B} T\right)^{2} e^{-h \omega c / k_{B} T}}{\left(1-e^{-h \omega c / k_{B} T}\right)^{2}} .
$$

The summation in (3) requires all fundamental frequencies $\left\{\omega_{i}\right\}$ of the molecular system to be computed in advance. Since these frequencies are related to the eigenvalues $\left\{\lambda_{i}\right\}$ of the Hessian matrix $F$ (second derivatives of the potential) by

$$
\omega=108.9 \sqrt{\bar{\lambda}},
$$


the heat capacity calculation ultimately amounts to computing the entire spectrum of $F$. Note that the above change of variable from $\omega$ to $\lambda$ does not alter the evaluation of the integrand. The integral defined by (1) is equivalent to

$$
C_{v}=\int_{0}^{\infty} H(108.9 \sqrt{\lambda}) \hat{g}(\lambda) d \lambda
$$

where $\hat{g}(\lambda) d \lambda$ gives the number of eigenvalues within the interval $(\lambda, \lambda+d \lambda)$.

For large-scale molecular systems consisting of hundreds of thousands of atoms, computing all eigenvalues of $F$ is very time-consuming. This difficulty leads to a commonly used approach of treating the system as a continuum [11]. Under this assumption, $\sigma(\omega)$ is considered a continuous and differentiable function (although it really isn't). As a result, $C_{v}$ can be calculated by some simple quadrature rule (e.g., the composite trapezoidal or Simpson's rule). A typical quadrature rule partitions the interval $\left[\omega_{1}, \omega_{n}\right]$ uniformly into a number of small subintervals $\left[\xi_{1}, \xi_{2}\right],\left[\xi_{2}, \xi_{3}\right]$, $\ldots,\left[\xi_{m-1}, \xi_{m}\right]$. The integrand is evaluated at two or three selected points within each subinterval; the results are then summed together following a particular rule specified by the quadrature. In this approach, $g(\omega)$ is no longer a sum of $\delta$-functions. Instead, it is often approximated by a finite difference formula

$$
g(\omega)=\frac{\sigma(\omega+\Delta \omega)-\sigma(\omega)}{\Delta \omega},
$$

where $\sigma(\omega)=\left|\left\{\omega_{i} \mid \omega_{i}<\omega\right\}\right|$. The notation $|\cdot|$ is used here to denote the number of elements contained in a set $\left\{\omega_{i}\right\}$. It follows from Sylvester's inertia theorem [12] that $\sigma(\omega)$ can be determined by decomposing the matrix $F-\omega I$ into a product of $L D L^{T}$, where $L$ is unit lower triangular and $D$ is diagonal. The number of negative entries on the diagonal of $D$ corresponds to $\sigma(\omega)$. Hence, the major cost of this algorithm is the computation required to perform a sequence of sparse $L D L^{T}$ factorizations. These factorizations must be carried out in a numerically stable manner using the Bunch and Kaufman [2] technique to ensure a reliable inertia count. It has been shown [14] that the computational procedure based on $L D L^{T}$ factorization and inertia count is considerably faster than the brute-force approach of computing all eigenvalues of $F$.

In this paper, we introduce another way of computing $C_{v}$. The new algorithm does not rely on treating the molecular system as a continuum. It approximates the Riemann-Stieltjes integral directly by a special Gaussian quadrature. No eigenvalue of $F$ is computed. Neither does the algorithm require any sparse matrix factorization. The Gaussian quadrature rule can be computed by a few steps of a Lanczos iteration. The main computational cost is reduced to that of a few sparse matrix vector multiplications. In the next section, we review Gaussian quadrature and point out its connection with orthogonal polynomials and the Lanczos process. In Section 4, we give a recipe for heat capacity calculation based on a special quadrature rule provided by a Lanczos iteration. We will also demonstrate the efficiency and accuracy of this calculation.

\section{Gaussian Quadrature}

The theory of Gaussian quadrature $[8,13,3]$ is closely related to the subject of orthogonal polynomials [7]. Under a particular Gaussian quadrature rule defined by a set of weights $\left\{\gamma_{i}\right\}$ and abscissas $\left\{\theta_{i}\right\}, i=1,2, \ldots, k$, the integration of a continuous function $f(t)$ with respect to a nonnegative 
measure $\mu(t)$ can be approximated by

$$
\int_{a}^{b} f(t) \mu(t) d t \approx \sum_{i=1}^{k} f\left(\theta_{i}\right) \gamma_{i}
$$

Each knot $\theta_{i}$ is a root of a $k$-th degree orthogonal polynomial $\phi_{k}$ associated with the inner product $\langle\cdot, \cdot\rangle$ defined below. The coefficients $\gamma_{i}$ 's are determined in a way so that the integral is exact for all polynomials with degree less than $2 k$.

For a continuous and nonnegative measure $\mu(t)$, the usual choice of the inner product for generating a sequence of orthogonal polynomials is

$$
\langle f, g\rangle=\int_{a}^{b} f(t) g(t) \mu(t) d t .
$$

For a discrete measure $\left\{\mu_{i}\right\}$ defined on $\left\{t_{i}\right\}, i=1,2, \ldots, n$, the above integral should be interpreted in Riemann-Stieltjes' sense, i.e.,

$$
\langle f, g\rangle=\sum_{i=1}^{n} f\left(t_{i}\right) g\left(t_{i}\right) \mu_{i} .
$$

Orthonormal polynomials can be generated via the Gram-Schmidt process or the following threeterm recurrence formula [7]

$$
\beta_{j+1} \phi_{j+1}(t)=\left(t-\alpha_{j}\right) \phi_{j}(t)-\beta_{j} \phi_{j-1}(t)
$$

where

$$
\alpha_{j}=\left\langle t \phi_{j-1}, \phi_{j-1}\right\rangle \text { and } \beta_{j}=\left\langle t \phi_{j-2}, \phi_{j-1}\right\rangle .
$$

It can be shown [7] that the tridiagonal Jacobi matrix

$$
J_{k}=\left(\begin{array}{cccc}
\alpha_{1} & \beta_{1} & & \\
\beta_{1} & \alpha_{2} & \ddots & \\
& \ddots & \ddots & \beta_{k-1} \\
& & \beta_{k-1} & \alpha_{k}
\end{array}\right)
$$

formed by placing $\alpha_{i}(i=1,2, \ldots, k)$ on the diagonal and $\beta_{i}(i=1,2, \ldots, k-1)$ on super- and subdiagonals, provides the abscissas $\left\{\theta_{i}\right\}$ and weights $\left\{\gamma_{i}\right\}$ needed in (5). The abscissas $\left\{\theta_{i}\right\}$ are simply the eigenvalues of $J_{k}$, and $\gamma_{i}=\tau_{i}^{2}$, where $\tau_{i}$ is the first entry of the eigenvector corresponding to $\theta_{i}$. These abscissas and weights essentially form a new distribution function

$$
\hat{\sigma}(\omega)=\sum_{i=1}^{k} \gamma_{i} h\left(\omega-\theta_{i}\right),
$$

that can be viewed as an approximation to (2). 


\section{Connection with the Lanczos Iteration}

The process of generating orthonormal polynomials associated with a nonnegative point measure defined on the spectrum of $F$ has a close connection with the Lanczos iteration [10]. Given an arbitrary nonzero starting vector $v_{0}$, the Lanczos process produces an orthonormal basis $\left\{v_{j}\right\}$ for the Krylov subspace

$$
\mathcal{K}\left(F, v_{0} ; k\right)=\operatorname{span}\left\{v_{0}, F v_{0}, F^{2} v_{0}, \ldots, F^{k-1} v_{0}\right\} .
$$

These basis vectors satisfy a three-term recurrence

$$
\beta_{j+1} v_{j+1}=F v_{j}-\alpha_{j} v_{j}-\beta_{j} v_{j-1},
$$

where

$$
\alpha_{j}=v_{j}^{T} F v_{j} \text { and } \beta_{j}=v_{j}^{T} F v_{j-1} \text {. }
$$

If we let

$$
V_{k}=\left(v_{1} v_{2} \ldots v_{k}\right), \quad f_{k}=\beta_{k} v_{k+1},
$$

and

$$
T_{k}=\left(\begin{array}{cccc}
\alpha_{1} & \beta_{1} & & \\
\beta_{1} & \alpha_{2} & \ddots & \\
& \ddots & \ddots & \beta_{k-1} \\
& & \beta_{k-1} & \alpha_{k}
\end{array}\right),
$$

the Lanczos processes can be succinctly described by the matrix equation

$$
F V_{k}=V_{k} T_{k}+f_{k} e_{k}^{T},
$$

where $e_{k}$ is an elementary unit vector with one in the $k$-th entry and zero elsewhere.

Since $v_{j} \in \mathcal{K}\left(F, v_{0} ; j\right), v_{j}=\phi_{j-1}(F) v_{0}$ for some $j-1$ st degree polynomial $\phi_{j-1}$. Suppose $\left\{\left(\lambda_{i}, z_{i}\right)\right\}, i=1,2, \ldots, n$, are eigenpairs of $F$ and $\Lambda=\operatorname{diag}\left(\lambda_{1}, \lambda_{2}, \ldots, \lambda_{n}\right)$. If $v_{0}=Z g$, where

$$
Z=\left(z_{1} z_{2} \ldots z_{n}\right) \text { and } g=\left(\sqrt{\mu_{1}} \sqrt{\mu_{2}} \ldots \sqrt{\mu_{n}}\right)^{T}
$$

then

$$
\begin{aligned}
v_{j}^{T} v_{i} & =\left(\phi_{j-1}(F) v_{0}\right)^{T}\left(\phi_{i-1}(F) v_{0}\right) \\
& =\left(\phi_{j-1}(F) Z g\right)^{T}\left(\phi_{i-1}(F) Z g\right) \\
& =g^{T} \phi_{j-1}(\Lambda) \phi_{i-1}(\Lambda) g \\
& =\sum_{\ell=1}^{n} \phi_{j-1}\left(\lambda_{\ell}\right) \phi_{i-1}\left(\lambda_{\ell}\right) \mu_{\ell} .
\end{aligned}
$$

The orthonormal condition

$$
v_{i}^{T} v_{j}= \begin{cases}1 & i=j \\ 0 & \text { otherwise }\end{cases}
$$

indicates that the Lanczos algorithm implicitly generates a sequence of orthonormal polynomials $\left\{\phi_{j}(\lambda)\right\}$ with respect to the point measure $\left\{\mu_{j}\right\}$. Moreover, the tridiagonal matrix $T_{k}$ that appears in (7) matches exactly with the Jacobi matrix (6). 


\section{Numerical Results}

The discussion above provides the theoretical basis for the following simple procedure that can be used to approximate the heat capacity of a large-scale molecular system.

1. Run a $k$-step $(k \ll n)$ Lanczos iteration

$$
F V_{k}=V_{k} T_{k}+f_{k} e_{k}^{T}
$$

2. Compute eigenvalues $\theta_{i}$ and eigenvectors of $T_{k}$. Set $\gamma_{i}=\tau_{i}^{2}$, where $\tau_{i}$ is the first entry the the $i$-th eigenvector. (The cost of this step can be further reduced by the technique of Golub and Welsh [9], which does not require all the eigenvectors of $T_{k}$ to be computed.)

3. Complete the Gaussian quadrature calculation

$$
C_{v} \approx \sum_{i=1}^{k} H\left(108.9 \sqrt{\theta_{i}}\right) \gamma_{i}
$$

A minor drawback of the above approach is that the point measure $\left\{\mu_{i}\right\}$ with which the integration is carried out is implicitly defined by the starting vector, $v_{0}$, of the Lanczos iteration. It is unlikely that this vector contains equal contributions from all eigenvectors of $F$. Nevertheless, when a random vector is chosen, all eigenvectors are likely to be well represented in $v_{0}$. The difference in measure may lead to different numerical values of $C_{v}$. However, the qualitative behavior of $C_{v}$ (e.g., its variation with respect to the temperature) can still be captured if the difference is negligibly small.

We applied the above procedure to PE particles of various sizes. For the 3000-atom particle, we compared Gaussian quadrature calculations with the exact integration obtained by

$$
C_{v}=\sum_{i=0}^{n} H\left(\omega_{i}\right) \mu_{i} .
$$

In order to obtain the point measure $\left\{\mu_{i}\right\}$, we must compute all the eigenvectors $\left\{z_{i}\right\}$ of $F$. If $Z=\left[\begin{array}{llll}z_{1} & z_{2} & \ldots & z_{n}\end{array}\right]$, then

$$
\mu_{i}=\left(Z^{T} v_{0}\right) e_{i}
$$

If Figure 2, we compare the distribution function $\sigma(\omega)$ associated with the original integral (1) with the approximation constructed by a 20-point Gaussian quadrature. We notice that the approximate distribution (dotted staircase line) closely captures the main features of the exact distribution function (solid curve).

As shown in Figure 3, the 20-point Gaussian quadrature constructed for the $3000 \mathrm{PE}$ particle gives fairly accurate approximation to $C_{v}$ at a wide range of temperatures. Notice that the difference between the exact $C_{v}$ calculated from (4) and the approximation obtained from (8) is negligible. As the number of atoms increases, a higher order Gaussian quadrature is needed. However, we found that for particles with as many as 24000 atoms, a 100-point quadrature is quite sufficient to provide the desired accuracy.

Figure 4 illustrates the results of two 20-point Gaussian quadrature calculations associated with two different measures. Both calculations were applied to the 3000-atom PE particle used in the 


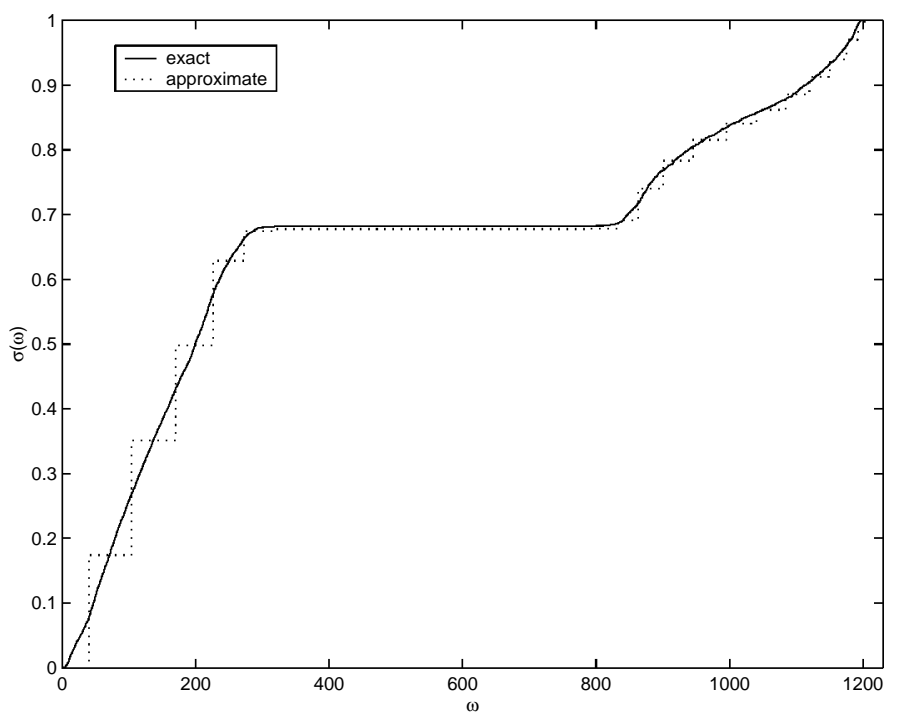

Figure 2: Distribution functions for a 3000-atom PE particle.

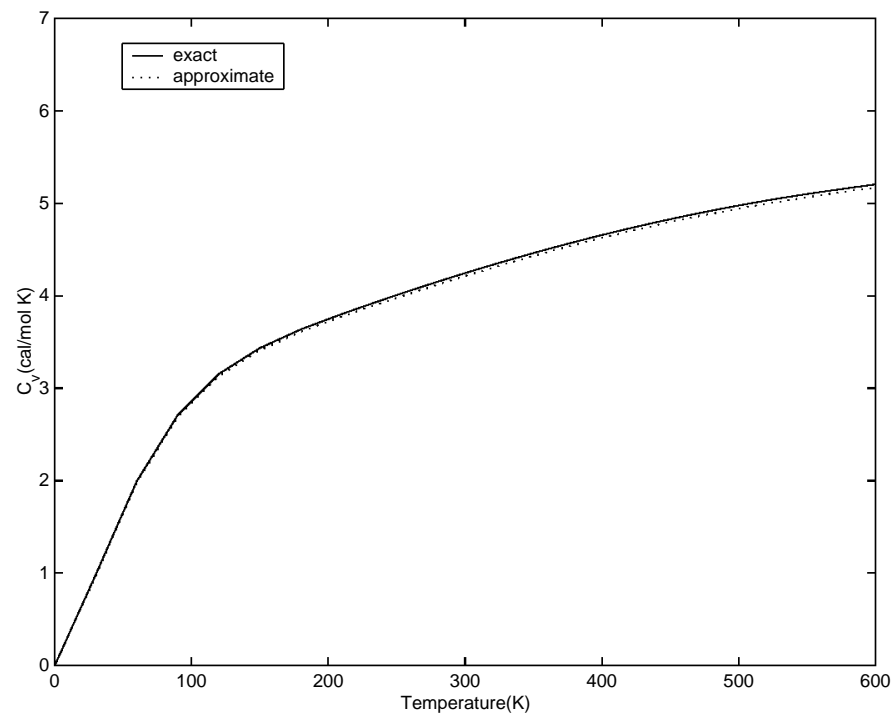

Figure 3: Exact and approximate $C_{v}$ for a 3000-atom PE particle at various temperatures. 
previous example. The solid curve corresponds to the calculation obtained from running a Lanczos iteration with $v_{0}=\sum_{i=1}^{n} z_{i} / \sqrt{n}$. The point measure associated with this calculation is $\mu_{i}=1 / n$. The dashed curve corresponds to a calculation obtained from a random starting vector. Clearly, the difference between these two calculations is negligibly small. Both calculations reveal the same variational pattern of $C_{v}$ with respect to the temperature.

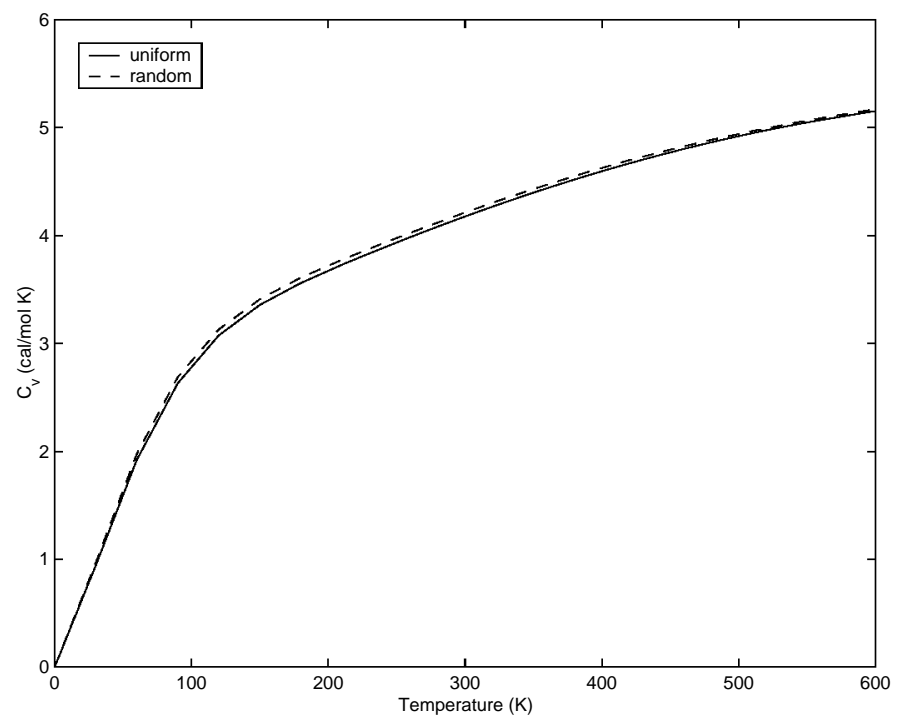

Figure 4: Approximate $C_{v}$ of a 3000-atom PE particle evaluated with two different measures.

As we mentioned earlier, the major cost of the quadrature calculation is the sparse matrix vector multiplication used in the Lanczos process. We also used Daniel, Gragg, Kaufman and Stewart's [4] reorthogonalizaton scheme to make sure that the Lanczos basis vectors $\left\{v_{i}\right\}$ are fully orthonormal. This guarantees that there is no loss of accuracy in the abscissas and weights used in the quadrature formula. The computational cost is clearly proportional to the number of atoms in the system and the number of Gaussian points used in the quadrature. Table 1 shows cost of heat capacity calculation in terms of CPU seconds. We use pe3k, pe6k and pe12k to denote polyethylene particles with 3000,6000 , and 12000 atoms respectively. The computation is done on a single processor of IBM SP3 (200 Mhz, 4MB cache). Notice that a 100-point Gaussian quadrature calculation for pe12k took a little over 100 seconds. This is at least a hundred times faster than our earlier approach [14] which took several hours.

\begin{tabular}{|c|c|c|c|}
\hline particle & 20 point & 50 point & 100 point \\
\hline \hline pe3k & 3.5 & 10.9 & 24.5 \\
\hline pe6k & 7.4 & 23.1 & 51.9 \\
\hline pe12k & 15.3 & 47.8 & 107.7 \\
\hline
\end{tabular}

Table 1: The cost of heat capacity calculation. 


\section{Conclusion}

We presented an algorithm for computing the heat capacity of a large-scale molecular system. The algorithm is based on a Gaussian quadrature rule specially designed for the Riemann-Stieljes integral (1). The abscissas and weights of the quadrature are computed from a Lanczos iteration. The main computational cost consists of a few sparse matrix vector multiplications. Numerical examples show that this method gives accurate results. We have also shown that this new algorithm

is considerably faster than earlier approaches that require either computing the full spectrum of the Hessian matrix or performing a sequence of $L D L^{T}$ factorizations.

\section{References}

[1] M. D. Barnes, K. C. Ng, K. Fukui, B. G. Sumpter, and D. W. Noid. Probing phase-separation behavior in polymer-blend microparticles: Effects of particle size and polymer mobility. Macromolecules, 32(21):7183-7189, 1999.

[2] J. R. Bunch and L. Kaufman. Some stable methods for calculating inertia and solving symmetric linear systems. Math. Comp., 31:162-179, 1977.

[3] D. Calvetti, G. H. Golub, W. B. Gragg, and L. Reichel. Computation of Gauss-Kronrod quadrature rules. Math. Comp., to appear, 2001.

[4] J. Daniel, W. B. Gragg, L. Kaufman, and G. W. Stewart. Reorthogonalization and stable algorithms for updating the Gram-Schmidt QR factorization. Math. Comp., 30, 1976.

[5] Bernd Fischer. Polynomial Based Methods for Symmetric Linear Systems. Wiley \& Teubner, Chichester, West Sussex, England, 1996.

[6] A. Friedman. Modern Analysis. Dover, New York, NY, 1980.

[7] G. Szegö. Orthogonal Polynomials. American Mathematical Society, Providence, RI, 1975.

[8] W. Gautschi. A survey of Gauss-Christoffel quadrature formulae. In P. Butzer and F. Fehér, editors, E. B. Christoffel: The influence of his work in mathematics and the physical sciences, pages 72-147. Birkhäuser, 1981.

[9] G. H. Golub and J. H. Welsh. Calculation of Gauss quadrature rules. Math. Comp., 23:61-76, 1969.

[10] C. Lanczos. An iteration method for the solution of the eigenvalue problem of linear differential and integral operators. J. Res. Nat. Bur. Stand., 45:255-281, 1950.

[11] D. A. McQuarrie. Statistical Mechanics. Harper \& Row, New York, NY, 1976.

[12] B. N. Parlett. The Symmetric Eigenvalue Problem. Prentice-Hall, Englewood Cliffs, NJ, 1980.

[13] A. H. Stroud and Don Secrest. Gaussian Quadrature Formulas. Prentice Hall, Englewood Cliffs, NJ, 1966. 
[14] C. Yang, K. Fukui, B. G. Sumpter, D. W. Noid, and R. E. Tuzun. Calculating the density of states for large-scale molecular systems. Macromolecular Theory and Simulations, 9:363-432, 2000 . 